\title{
Composição florística e estrutura fitossociológica de áreas de campo sujo e cerrado sentido restrito na Floresta Nacional de Silvânia, Goiás, Brasil
}

\author{
Walter Santos de Araújo ${ }^{1}$, Éder Dasdoriano Porfírio Júnior ${ }^{1}$, \\ Augusto Francener ${ }^{2,3}$, Climbiê Ferreira Hall ${ }^{2,3}$
}

Enviado em junho de 2012; aceito em novembro de 2012.

\section{Resumo}

O Cerrado é a savana com maior riqueza florística do planeta e também uma das regiões mais fragmentadas e ameaçadas do mundo. Diante disso, inventariar e conhecer a diversidade de espécies vegetais no Cerrado uma iniciativa muito importante. O presente trabalho foi realizado na Floresta Nacional de Silvânia, com o objetivo de analisar a estrutura fitossociológica das vegetações de cerrado sentido restrito (cerrado s.r.) e campo sujo da unidade de conservação. A amostragem foi realizada através de parcelas de $10 \times 10 \mathrm{~m}$, sendo 15 no cerrado s.r. e 10 no campo sujo. Entraram na amostragem os indivíduos lenhosos com diâmetro, igual ou superior a $5 \mathrm{~cm}$ a $30 \mathrm{~cm}$ do solo. Foram registradas 60 espécies em 46 gêneros distribuídas em 28 famílias, sendo 52 espécies no cerrado s.r. e 27 no campo sujo. As famílias que apresentaram mais espécies foram Malpighiaceae (7), Fabaceae (6) e Vochysiaceae (5). As espécies com maior Índice de Valor de Importância foram Qualea grandiflora, Kielmeyera coriacea e Styrax ferrugineus para o cerrado s.r., e $S$. ferrugineus, Piptocarpha rotundifolia e Erythroxylum deciduum para o campo sujo. No cerrado s.r. a densidade e área basal foram de 1125 indivíduos/ha e $5,32 \mathrm{~m}^{2} / \mathrm{ha}$ respectivamente. Já no campo sujo foram 730 indivíduos/ha e $1,96 \mathrm{~m}^{2} / \mathrm{ha}$. O índice de Shannon $\left(\mathrm{H}^{\prime}\right)$ encontrado para o cerrado s.r. foi de 3,48, e para o campo sujo 2,91, valores que evidenciam uma alta diversidade de espécies para a área em estudo.

\footnotetext{
DOI: http://dx.doi.org/10.5007/2178-4574.2012n41p42

${ }^{1}$ Departamento de Ecologia, Instituto de Pesquisas Ambientais e Ações Conservacionistas, 74025-020, Centro, Goiânia, GO, Brasil. walterbioaraujo@yahoo.com.br

${ }^{2}$ Laboratório de Morfologia e Taxonomia Vegetal, Departamento de Biologia Geral, Instituto de Ciências Biológicas, Universidade Federal de Goiás, Caixa Postal 131, 74001-970, Campus Samambaia, Goiânia, GO, Brasil.

${ }^{3}$ Instituto de Botânica de São Paulo, Av. Miguel Stéfano 3687, 04301-902, Vila Água Funda, São Paulo, SP, Brasil.
}

\section{(cc) $\mathrm{BY}-\mathrm{NC}$}

Este artigo é de Acesso Livre, disponibilizado sob os termos da

Creative Commons Attribution 3.0 Unported License (http://creativecommons.org/licenses/by/3.0/) que permite uso não-comercial, distribuição e reprodução em qualquer meio, desde que este trabalho original seja devidamente citado. 
Palavras-Chave: Cerrado, diversidade florística, estrutura vegetacional, FLONA.

\begin{abstract}
(Floristic composition and phytosociological structure of the grassland and cerrado areas in the Floresta Nacional de Silvânia, Goiás, Brazil) The Cerrado is the savanna with largest floristic richness of planet and also one of the most fragmented and threatened regions of world. Therefore, inventory and know the diversity of plant species in the Cerrado is a very important initiative. This study was conducted at the Floresta Nacional de Silvânia, aiming to investigate the phytosociological structure of the "cerrado sensu stricto" ("cerrado s.r.") and "campo sujo" vegetations. Sampling was performed in plots of 10 x 10 m, 15 in the "cerrado s.r." and 10 in the "campo sujo". Were sampled only woody individuals with a diameter equal to, or greater than $5 \mathrm{~cm}$ (30 cm from the soil). Results showed 60 species belonging to 28 families and 46 genera, 52 species in the "cerrado s.r." and 27 in the "campo sujo'. The families with more species were Malpighiaceae (7), Fabaceae (6) and Vochysiaceae (5). The species with the highest importance values in the "cerrado s.r." were Qualea grandiflora, Kielmeyera coriacea, and Styrax ferrugineus, on the other hand, in the "campo sujo" were S. ferrugineus, Piptocarpha rotundifolia e Erythroxylum deciduum. In the "cerrado s.r.", density and basal area were 1125 individuals/ha and $5.32 \mathrm{~m}^{2} /$ ha respectively. In the "campo sujo" were 730 indivíduos/ha and $1.96 \mathrm{~m}^{2} / \mathrm{ha}$. The Shannon index $\left(\mathrm{H}^{\prime}\right)$ estimated for the "cerrado s.r." was 3.48, and for the "campo sujo" 2.91 . These values demonstrate a high diversity of species in the study area.
\end{abstract}

Key words: Cerrado, FLONA, floristic diversity, vegetational structure.

\title{
Introdução
}

O Cerrado é o segundo maior bioma brasileiro ocupando uma área de 2 milhões de $\mathrm{km}^{2}$, o que corresponde a $23 \%$ do território nacional, abrangendo estados em todas as regiões do país (Oliveira \& Ratter 2002). Esse bioma é formado por diversos tipos de formações vegetais, tornando-se um dos mais ricos biomas do Brasil e do mundo (Klink \& Machado 2005).

Segundo Ribeiro \& Walter (1998) o Cerrado apresenta três grandes grupos de vegetações: campestres, savânicas e florestais. As formações campestres são constituídas pelo campo limpo, campo sujo e campo rupestre. Dentre as formações savânicas encontram-se as fitofisionomias de cerrado rupestre e cerrado sentido restrito (cerrado s.r.), que é a fitofisionomia característica do bioma. Já as formações florestais são constituídas pelo cerradão, mata de interflúvio, mata ciliar e mata de galeria.

O Cerrado abriga algo entre 20 e $50 \%$ da biodiversidade brasileira, dependendo do grupo de organismos avaliado, sendo considerado um dos biomas prioritários para conservação no planeta (Myers et al. 2000; Klink \& Machado 2005). 
A fragmentação e a perda de habitat têm causado perda de diversidade e colocado em risco de extinção muitas espécies do bioma (Klink \& Machado 2005). Segundo Cunha et al. (2007), cerca de $21,87 \%$ da área remanescente de vegetação nativa de Cerrado no Estado de Goiás é constituída por cerrado s.r., já as fitofisionomias campestres abrigam apenas $8,76 \%$. Segundo eles, das áreas remanescentes, mais de $82 \%$ apresentam tamanho menor do que 1 ha. Esses dados indicam que além de poucas áreas naturais, as existentes estão muito fragmentadas e desconexas.

Sob o ponto de vista florístico o bioma apresenta mais de 12.000 espécies de plantas vasculares (Mendonça et al. 2008), e muitas delas vêm sofrendo grande ameaça (Ratter et al. 1997). Estudos fitossociológicos têm fornecido informações importantes para a compreensão dos padrões biogeográficos do Cerrado, e subsidiado a determinação de áreas prioritárias para a conservação (Felfili et al. 2002). Padrões florísticos e estruturais evidenciados por esse tipo de estudo, como variação na densidade, riqueza e diversidade-beta, entre diferentes áreas, são boas ferramentas para elaborar estratégias de conservação ao nível local e regional (Felfili et al. 2004).

A Floresta Nacional de Silvânia é a única unidade de conservação federal da região central do estado de Goiás, sendo que existem poucos registros na literatura sobre a fauna e, principalmente sobre a flora da região. Recentemente Francener $e$ t al. (2012) realizaram o inventário florístico da unidade e entorno registrando um total de 244 espécies de angiospermas (67 famílias e 177 gêneros). Apesar da importância dessa contribuição, ainda não há registros na literatura de estudos que investiguem aspectos da estrutura vegetacional da unidade. Desse modo, o objetivo do presente trabalho foi realizar o levantamento florístico e fitossociológico da comunidade arbóreo-arbustiva de fitofisionomias de campo sujo e cerrado s.r. da Floresta Nacional de Silvânia.

\section{Material e Métodos Área de Estudo}

A Floresta Nacional de Silvânia (FLONA-Silvânia) localiza-se no município de Silvânia (Figura 1), a 95 km de Goiânia, na Mesorregião Leste do Estado de Goiás (16039'26'S e 48 36'16'). Dentro da unidade existem algumas famílias que residem fazendo uso sustentável dos recursos naturais, bem como são desenvolvidas diversas atividades de pesquisa (Francener et al. 2012). A FLONA-Silvânia apresenta uma área de 466,55 ha e uma altitude média de $900 \mathrm{~m}$. O clima da região é classificado como Aw de Köppen (tropical chuvoso), com verão quente e chuvoso (outubro a março) e inverno seco e frio (abril a setembro). $\mathrm{Na}$ unidade são encontrados vários tipos de vegetação que compõem o bioma Cerrado, tais como: campo sujo, cerrado s.r., cerradão, floresta de galeria, floresta mesofítica de interflúvio e vereda (Francener et al. 2012). O levantamento fitossociológico foi realizado entre março e maio de 2010 em fitofisionomias de campo sujo e cerrado s.r. presentes na FLONASilvânia. 
A

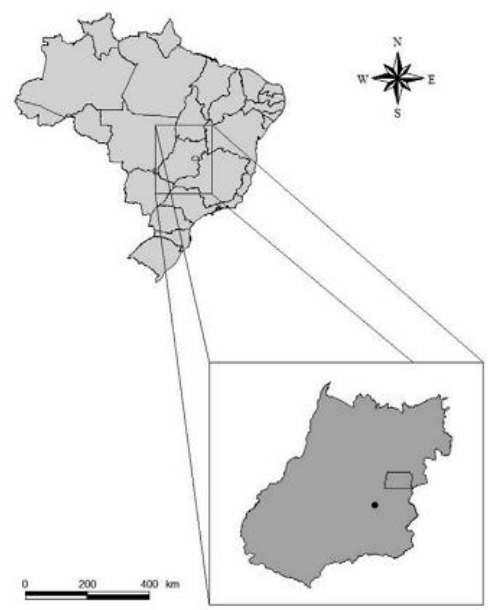

B

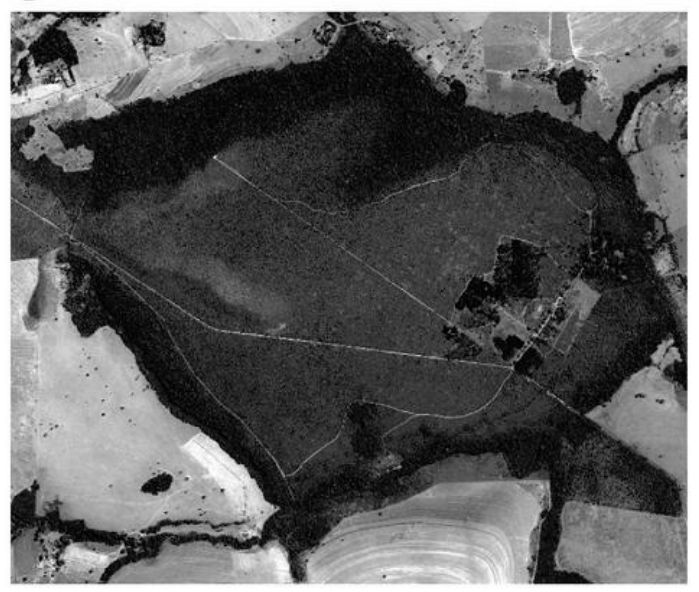

Figura 1. (A) Localização do município de Silvânia, estado de Goiás, Brasil. (B) Floresta Nacional de Silvânia (FLONA-SILVÂNIA), estado de Goiás, Brasil. Modificado de Morais $e t$ al. (2012).

\section{Amostragem}

A amostragem foi realizada através de parcelas de 10 × $10 \mathrm{~m}(0,01 \mathrm{ha})$, sendo 15 no cerrado s.r. e 10 no campo sujo (totalizando 0,25 ha amostrados). $\mathrm{O}$ número menor de parcelas amostradas no campo sujo foi devido a pequena área de cobertura dessa fitofisionomia na unidade. Em cada parcela foram incluídos todos os indivíduos arbóreo-arbustivos com diâmetro igual ou superior a $5 \mathrm{~cm}$ a $30 \mathrm{~cm}$ do solo. Dos indivíduos amostrados foram coletados ramos, geralmente com estruturas reprodutivas, sendo os exemplares herborizados e depositados na coleção do Instituto de Pesquisas Ambientais e Ações Conservacionistas - IPAAC (Goiânia, GO). A identificação dos espécimes foi realizada através de literatura especializada (e.g. Lorenzi 1992; Silva Júnior 2005), comparação com amostras do herbário UFG da Universidade Federal de Goiás, e consultas a especialistas. Para a classificação em família utilizou-se o sistema The Angiosperm Phylogeny Group (2009).

\section{Análise fitossociológica}

A partir dos dados coletados em campo foram calculados os seguintes parâmetros fitossociológicos: densidade absoluta (DA), freqüência absoluta (FA), dominância absoluta (DoA) e os respectivos parâmetros relativos (densidade, freqüência e dominância relativas) (Felfili \& Venturoli 2000). Com base nos parâmetros relativos, foram calculados o Índice de Valor de Importância (IVI) e o Índice de Valor de Cobertura (IVC) para cada espécie. Foram calculados também o Índice de Diversidade de Shannon $\left(\mathrm{H}^{\prime}\right)$ e o Índice de Equatabilidade de Pielou $\left(\mathrm{J}^{\prime}\right)$ 
(Felfili \& Venturoli 2000). Além disso, para análise dos padrões de estratificação horizontal foram estabelecidas quatro classes de diâmetro: Classe 1: $<6 \mathrm{~cm}$; Classe 2: 6,1 - $8 \mathrm{~cm}$; Classe 3: 8,1-10 cm e Classe $4>10 \mathrm{~cm}$. Ficou estabelecido também o mesmo número de classes para a análise da estratificação vertical, com a distribuição das alturas em intervalos de classe de 1 a 4 (Classe 1: $<2 \mathrm{~m}$, Classe 2: $2,1-3 \mathrm{~m}$, Classe 3: 3,1 - 4,9 m e Classe 4: > $5 \mathrm{~m}$ ). A frequência de indivíduos entre as diferentes classes de diâmetro e altura foram comparadas através do teste não paramétrico de Qui-Quadrado no programa Statistica 7.0.

\section{Resultados e Discussão}

Foram registradas 60 espécies de plantas lenhosas distribuídas em 28 famílias e 46 gêneros, sendo 52 espécies no cerrado s.r. e 27 no campo sujo (Tabela 1). Destas, 33 ocorreram exclusivamente no cerrado s.r. e oito no campo sujo. As famílias melhor representadas em número de espécies foram Malpighiaceae (7), Fabaceae (6) e Vochysiaceae (5).

As famílias com maior IVI no campo sujo foram Styracaceae, Asteraceae e Bignoniaceae, com 65,6, 33,0 e 26,1, respectivamente (Figura 2a). Elas abrigaram $41,5 \%$ do IVI e 53,1\% da área basal da comunidade. Para o cerrado s.r., Vochysiaceae, Clusiaceae e Malpighiaceae foram as famílias mais importantes. Com IVI de 48,9, 37,5 e 29,7, respectivamente, elas representam 38,7\% do IVI total e $41,3 \%$ da área basal da fitofisionomia (Figura 2b). Algumas dessas famílias com maior IVI estão listadas entre as 10 famílias com maior número de espécies registradas para o bioma Cerrado, que são: Fabaceae, Asteraceae, Orchidaceae, Poaceae, Rubiaceae, Melastomataceae, Myrtaceae, Euphorbiaceae, Malpighiaceae e Lythraceae (Mendonça et al. 2008).

Vochysiaceae, que foi a família mais representativa nas fitofisionomias amostradas da FLONA-Silvânia, apresentou os maiores IVI’s em levantamentos fitossociológicos em áreas de cerrado s.r. na Reserva Biológica do IBGE (Andrade et al. 2002) e no Município de Água Boa (Felfili et al. 2002), ambas no Distrito Federal. No presente estudo, Fabaceae também foi particularmente importante nas fitofisionomias estudadas sendo a segunda mais diversa no cerrado s.r. (6) e a mais rica campo sujo (3). Além disso, ela foi a quarta família mais importante em ambas as fitofisionomias com IVI de 22,4 e 22,9, para o cerrado s.r. e o campo sujo, respectivamente (Figura 2a e 2b). Fabaceae é a família mais rica do Cerrado e uma das mais comuns em levantamentos nesse bioma (Mendonça et al. 2008).

Das famílias que apresentaram maior IVI nesse estudo, Styracaceae no campo sujo e Vochysiaceae no cerrado s.r., nenhuma é citada entre as 10 famílias mais representativas do Cerrado (Mendonça et al. 2008). Tal resultado para essas famílias na FLONA-Silvânia se deve em particular à grande abundância de Styrax ferrugineus Nees \& Mart. (Styracaceae) e Qualea grandiflora Mart. (Vochysiaceae), que foram as espécies mais importantes no campo sujo e no cerrado s.r., respectivamente. 
Tabela 1. Lista de famílias e espécies lenhosas encontradas na Floresta Nacional de Silvânia, Goiás, Brasil.

\begin{tabular}{|c|c|c|c|c|}
\hline Família & Espécie & Nome Popular & Cerrado & $\begin{array}{c}\text { Campo } \\
\text { Sujo }\end{array}$ \\
\hline \multirow[t]{3}{*}{ Apocynaceae } & Aspidosperma macrocarpa Mart. & Guatambu & $\mathrm{X}$ & \\
\hline & Aspidosperma tomentosum Mart. & Peroba & $X$ & \\
\hline & Hancornia speciosa Gomes & Mangaba & $\mathrm{X}$ & \\
\hline \multirow[t]{2}{*}{ Araliaceae } & $\begin{array}{l}\text { Schefflera macrocarpa (Cham. \& } \\
\text { Schltdl.) Frodin }\end{array}$ & $\begin{array}{l}\text { Mandiocão-do- } \\
\text { cerrado }\end{array}$ & $\mathrm{X}$ & $\mathrm{X}$ \\
\hline & $\begin{array}{l}\text { Schefflera vinosa (Cham. \& } \\
\text { Schltdl.) Frodin \& Fiaschi }\end{array}$ & Mandiocão & $\mathrm{X}$ & \\
\hline \multirow[t]{2}{*}{ Asteraceae } & $\begin{array}{l}\text { Piptocarpha rotundifolia (Less.) } \\
\text { Baker. }\end{array}$ & Coração-de-negro & $\mathrm{X}$ & $\mathrm{X}$ \\
\hline & Asteraceae sp. & - & $\mathrm{X}$ & \\
\hline \multirow[t]{3}{*}{ Bignoniaceae } & $\begin{array}{l}\text { Tabebuia aurea (Silva Manso) } \\
\text { Benth. \& Hook. f.ex S.Moore }\end{array}$ & Caraíba & $\mathrm{X}$ & $\mathrm{X}$ \\
\hline & $\begin{array}{l}\text { Handroanthus ochraceus (Cham.) } \\
\text { Mattos }\end{array}$ & $\begin{array}{l}\text { Ipê amarelo-do- } \\
\text { cerrado }\end{array}$ & $\mathrm{X}$ & $\mathrm{X}$ \\
\hline & Zeyhera montana Mart. & Bolsinha-de-pastor & $X$ & \\
\hline Caryocaraceae & Caryocar brasiliense Cambess. & Pequi & $\mathrm{X}$ & \\
\hline Celastraceae & Plenckia populnea Reissek & Mangabeira-brava & $\mathrm{X}$ & \\
\hline \multirow[t]{2}{*}{ Clusiaceae } & Kielmeyera coriacea Mart. \& Zucc. & Pau-santo & $\mathrm{X}$ & $\mathrm{X}$ \\
\hline & Clusiaceae sp. & - & $\mathrm{X}$ & \\
\hline Combretaceae & Terminalia argentea Mart. & Capitão-do-campo & $\mathrm{X}$ & $X$ \\
\hline Connaraceae & Connarus suberosus Planch. & Arariba-do-campo & $\mathrm{X}$ & \\
\hline Dilleniaceae & Davilla elliptica A.St.-Hil. & Lixeirinha & $X$ & $\mathrm{X}$ \\
\hline Ebenaceae & Diospyros hispida A.DC. & Caqui-do-mato & $\mathrm{X}$ & \\
\hline \multirow[t]{4}{*}{ Erythroxylaceae } & Erythroxylum deciduum A.St.-Hil. & Mercúrio-do-campo & & $\mathrm{X}$ \\
\hline & Erythroxylum suberosum A.St.-Hil. & Mercúrio-do-campo & $X$ & \\
\hline & Erythroxylum tortuosum Mart. & Muxiba-comprida & $\mathrm{X}$ & \\
\hline & Erythroxylum sp. & Mercúlio-do-campo & $\mathrm{X}$ & $\mathrm{X}$ \\
\hline \multirow[t]{6}{*}{ Fabaceae } & Andira paniculata Benth. & Angelim & $X$ & \\
\hline & Dimorphandra mollis Benth. & Faveiro & $\mathrm{X}$ & $\mathrm{X}$ \\
\hline & Leptolobium dasycarpum Vogel & Amargosinha & $\mathrm{X}$ & $\mathrm{X}$ \\
\hline & Machaerium acutifolium Vogel & Jacarandá-do-cerrado & $X$ & \\
\hline & Machaerium opacum Vogel & Jacarandá-do-cerrado & $\mathrm{X}$ & \\
\hline & Sclerolobium paniculatum Vogel & Carvoeiro & $\mathrm{X}$ & $\mathrm{X}$ \\
\hline
\end{tabular}




\begin{tabular}{|c|c|c|c|c|}
\hline \multirow[t]{2}{*}{ Lauraceae } & Ocotea sp. & Canela & & $\mathrm{X}$ \\
\hline & Lauraceae sp. & - & $\mathrm{X}$ & \\
\hline \multirow[t]{7}{*}{ Malpighiaceae } & Banisteriopsis goiana B. Gates & - & $X$ & \\
\hline & Byrsonima coccolobifolia Kunth & Murici-rosa & $X$ & $\mathrm{X}$ \\
\hline & Byrsonima pachyphylla A.Juss. & Murici & $X$ & $\mathrm{X}$ \\
\hline & Byrsonima verbascifolia (L.) DC. & Muricizão & $X$ & \\
\hline & Byrsonima sp. & Murici & $\mathrm{X}$ & \\
\hline & Heteropterys byrsonimifolia A.Juss. & Murici-macho & $X$ & \\
\hline & Heteropterys sp. & - & $X$ & \\
\hline Malvaceae & $\begin{array}{l}\text { Pseudobombax longiflorum (Mart. } \\
\text { \& Zucc.) A.Robyns. }\end{array}$ & Embiruçu & & $\mathrm{X}$ \\
\hline \multirow[t]{2}{*}{ Melastomataceae } & Miconia sp. & Miconia & $X$ & \\
\hline & Tibouchina sp. & Quaresmeira & & $\mathrm{X}$ \\
\hline \multirow[t]{2}{*}{ Myrsinaceae } & Myrsinaceae sp. & - & $X$ & \\
\hline & Myrsine sp. & - & $X$ & \\
\hline \multirow[t]{3}{*}{ Myrtaceae } & $\begin{array}{ll}\text { Campomanesia } & \text { adamantium } \\
\text { (Cambess.) O.Berg } & \end{array}$ & Gabiroba & $X$ & \\
\hline & Myrcia bella Cambess. & Myrcia & $\mathrm{X}$ & \\
\hline & Psidium sp. & Araçá & & $\mathrm{X}$ \\
\hline \multirow[t]{2}{*}{ Nyctaginaceae } & Guapira noxia (Netto) Lundell & Capa-rosa & $X$ & $\mathrm{X}$ \\
\hline & Neea theifera Oerst. & - & $X$ & \\
\hline Ochnaceae & $\begin{array}{l}\text { Ouratea hexasperma (A.St.-Hil.) } \\
\text { Baill. }\end{array}$ & Vassoura-de-bruxa & $X$ & \\
\hline Proteaceae & Roupala montana Aubl. & Carne-de-vaca & $X$ & $\mathrm{X}$ \\
\hline Rubiaceae & Palicourea rigida Kunth & Chapéu-de-couro & $X$ & \\
\hline Sapindaceae & Sapindaceae sp. & - & & $\mathrm{X}$ \\
\hline Styracaceae & Styrax ferrugineus Nees \& Mart. & $\begin{array}{l}\text { Laranjinha-do- } \\
\quad \text { cerrado }\end{array}$ & $X$ & $\mathrm{X}$ \\
\hline Velloziaceae & Vellozia sp. & Canela-de-ema & $X$ & \\
\hline \multirow[t]{5}{*}{ Vochysiaceae } & Qualea grandiflora Mart. & Pau-terra-grande & $X$ & $\mathrm{X}$ \\
\hline & Qualea parviflora Mart. & Pau-terra-pequeno & $X$ & $\mathrm{X}$ \\
\hline & Qualea sp. & Pau-terra & $X$ & \\
\hline & $\begin{array}{l}\text { Salvertia convallariodora A.St.- } \\
\text { Hil. }\end{array}$ & Chapéu-de-couro & $X$ & \\
\hline & Vochysia sp. & - & & $\mathrm{X}$ \\
\hline Indeterminada & Indeterminada & - & $X$ & $\mathrm{X}$ \\
\hline
\end{tabular}



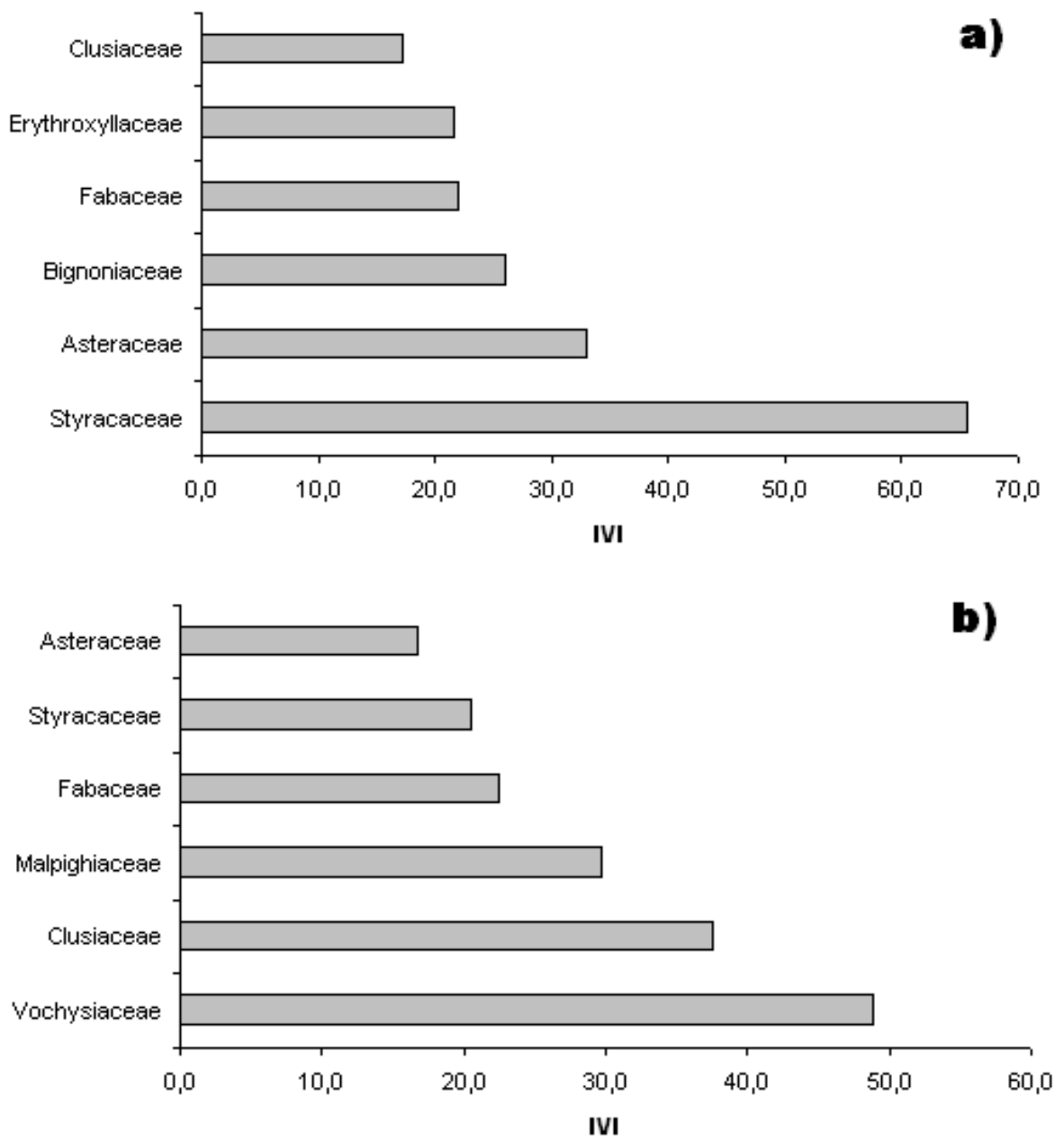

Figura 2. Distribuição dos IVI's por famílias de plantas nas fitofisionomias de a) campo sujo e b) cerrado sentido restrito da Floresta Nacional de Silvânia, Goiás, Brasil.

Os gêneros que apresentaram maior número de espécies foram Byrsonima (4), Erythroxylum (4) e Qualea (3) para ambas as fitofisionomias. As espécies mais representativas registradas no cerrado s.r. foram $Q$. grandiflora, Kielmeyera coriacea (Spr) Mart. e $S$. ferrugineus, com IVI de 34,7, 32,4 e 19,0, respectivamente (Tabela 2). Essas três espécies representaram $28,7 \%$ do IVI e 34,5\% da área basal da comunidade. Para o campo sujo, as espécies que apresentaram maior IVI foram $S$. 
ferrugineus, Piptocarpha rotundifolia (Less.) Baker. e Erythroxylum deciduum A.St.Hil., com 65,4, 32,8 e 17,3, respectivamente, representando juntas quase $40 \%$ do IVI da comunidade vegetal dessa fitofisionomia (Tabela 3). Esses elevados valores de IVI atribuídos a essas poucas espécies demonstram que, tanto no campo sujo quanto no cerrado s.r., a comunidade é caracterizada pela existência de poucas espécies dominantes e muitas raras e de pequena participação na ocupação da área (Andrade $e t$ al. 2002; Assunção \& Felfili 2004).

Qualea grandiflora foi registrada com o segundo maior IVI em uma área de cerrado s.r. no Parque Estadual da Serra de Caldas Novas, GO, onde $K$. coriacea foi a espécie mais importante (Silva et al. 2002). Na comunidade arbórea do serrado sentido restrito da APA do Paranoá, DF, a espécie $S$. ferrugineus teve o segundo maior IVI e $P$. rotundifolia teve o décimo (Assunção \& Felfili 2004). Moura et al. (2007) listam $S$. ferrugineus como a décima espécie com maior IVI para o cerrado rupestre do Parque Estadual da Serra dos Pireneus, GO.

Tabela 2. Parâmetros fitossociológicos das espécies arbóreo-arbustivas de cerrado sentido restrito na Floresta Nacional de Silvânia, Goiás, Brasil.

\begin{tabular}{|c|c|c|c|c|c|c|c|c|c|c|}
\hline Espécie & NI & $\mathbf{A B}\left(\mathbf{m}^{2}\right)$ & NP & DR & DoR & FA & FR & IVC & IVI & $\begin{array}{l}\% \\
\text { IVI }\end{array}$ \\
\hline Qualea grandiflora & 15 & 0,966 & 10 & 8,3 & 18,2 & 62,5 & 8,2 & 26,5 & 34,7 & 11,6 \\
\hline Kielmeyera coriacea & 24 & 0,534 & 11 & 13,3 & 10,0 & 68,8 & 9,0 & 23,4 & 32,4 & 10,8 \\
\hline Styrax ferrugineus & 12 & 0,350 & 7 & 6,7 & 6,6 & 43,8 & 5,7 & 13,2 & 19,0 & 6,3 \\
\hline Piptocarpha rotundifolia & 9 & 0,280 & 5 & 5,0 & 5,3 & 31,3 & 4,1 & 10,3 & 14,4 & 4,8 \\
\hline Byrsonima pachyphylla & 9 & 0,164 & 7 & 5,0 & 3,1 & 43,8 & 5,7 & 8,1 & 13,8 & 4,6 \\
\hline Connarus suberosus & 9 & 0,291 & 3 & 5,0 & 5,5 & 18,8 & 2,5 & 10,5 & 12,9 & 4,3 \\
\hline Tabebuia aurea & 5 & 0,276 & 3 & 2,8 & 5,2 & 18,8 & 2,5 & 8,0 & 10,4 & 3,5 \\
\hline Qualea parviflora & 7 & 0,179 & 3 & 3,9 & 3,4 & 18,8 & 2,5 & 7,3 & 9,7 & 3,2 \\
\hline Schefflera vinosa & 6 & 0,119 & 4 & 3,3 & 2,2 & 25,0 & 3,3 & 5,6 & 8,9 & 3,0 \\
\hline Erythroxylum suberosum & 6 & 0,140 & 3 & 3,3 & 2,6 & 18,8 & 2,5 & 6,0 & 8,4 & 2,8 \\
\hline Sclerolobium paniculatum & 1 & 0,287 & 1 & 0,6 & 5,4 & 6,3 & 0,8 & 6,0 & 6,8 & 2,3 \\
\hline Dimorphandra mollis & 4 & 0,063 & 4 & 2,2 & 1,2 & 25,0 & 3,3 & 3,4 & 6,7 & 2,2 \\
\hline Aspidosperma macrocarpa & 5 & 0,072 & 3 & 2,8 & 1,4 & 18,8 & 2,5 & 4,1 & 6,6 & 2,2 \\
\hline Miconia sp. & 3 & 0,139 & 2 & 1,7 & 2,6 & 12,5 & 1,6 & 4,3 & 5,9 & 2,0 \\
\hline $\begin{array}{l}\text { Heteropterys } \\
\text { byrsonimifolia }\end{array}$ & 4 & 0,063 & 3 & 2,2 & 1,2 & 18,8 & 2,5 & 3,4 & 5,9 & 2,0 \\
\hline Asteraceae sp. & 3 & 0,067 & 3 & 1,7 & 1,3 & 18,8 & 2,5 & 2,9 & 5,4 & 1,8 \\
\hline Byrsonima coccolobifolia & 3 & 0,030 & 3 & 1,7 & 0,6 & 18,8 & 2,5 & 2,2 & 4,7 & 1,6 \\
\hline Byrsonima sp. & 3 & 0,072 & 2 & 1,7 & 1,4 & 12,5 & 1,6 & 3,0 & 4,7 & 1,6 \\
\hline Qualea sp. & 4 & 0,041 & 2 & 2,2 & 0,8 & 12,5 & 1,6 & 3,0 & 4,6 & 1,5 \\
\hline
\end{tabular}


Tabela 2. Continuação

Terminalia argentea

Lauraceae sp.

Ouratea hexasperma

Banisteriopsis goiana

Plenckia populnea

Byrsonima verbascifolia

Machaerium acutifolium

Handroanthus ochraceus

Machaerium opacum

Leptolobium dasycarpum

Erythroxylum tortuosum

Schefflera macrocarpa

Davilla elliptica

Caryocar brasiliense

Hancornia speciosa

Myrsine sp.

Diospyros hispida

Guapira noxia

Clusiaceae sp.

Andira paniculata

Vellozia sp.

Heteropterys sp.

Salvertia convallariodora

Campomanesia

adamantium

Aspidosperma tomentosum

Myrcia bella

Erythroxylum sp.

Indeterminada

Zeyhera montana

Myrsinaceae sp.

Roupala montana

Neea theifera

Palicourea rigida

$\begin{array}{llllllllll}2 & 0,132 & 1 & 1,1 & 2,5 & 6,3 & 0,8 & 3,6 & 4,4 & 1,5 \\ 2 & 0,083 & 2 & 1,1 & 1,6 & 12,5 & 1,6 & 2,7 & 4,3 & 1,4 \\ 2 & 0,072 & 2 & 1,1 & 1,3 & 12,5 & 1,6 & 2,5 & 4,1 & 1,4 \\ 3 & 0,040 & 2 & 1,7 & 0,8 & 12,5 & 1,6 & 2,4 & 4,1 & 1,4 \\ 1 & 0,126 & 1 & 0,6 & 2,4 & 6,3 & 0,8 & 2,9 & 3,7 & 1,2 \\ 2 & 0,087 & 1 & 1,1 & 1,6 & 6,3 & 0,8 & 2,8 & 3,6 & 1,2 \\ 3 & 0,057 & 1 & 1,7 & 1,1 & 6,3 & 0,8 & 2,7 & 3,6 & 1,2 \\ 2 & 0,038 & 2 & 1,1 & 0,7 & 12,5 & 1,6 & 1,8 & 3,5 & 1,2 \\ 2 & 0,028 & 2 & 1,1 & 0,5 & 12,5 & 1,6 & 1,6 & 3,3 & 1,1 \\ 2 & 0,021 & 2 & 1,1 & 0,4 & 12,5 & 1,6 & 1,5 & 3,1 & 1,0 \\ 2 & 0,021 & 2 & 1,1 & 0,4 & 12,5 & 1,6 & 1,5 & 3,1 & 1,0 \\ 2 & 0,018 & 2 & 1,1 & 0,3 & 12,5 & 1,6 & 1,5 & 3,1 & 1,0 \\ 2 & 0,016 & 2 & 1,1 & 0,3 & 12,5 & 1,6 & 1,4 & 3,1 & 1,0 \\ 1 & 0,060 & 1 & 0,6 & 1,1 & 6,3 & 0,8 & 1,7 & 2,5 & 0,8 \\ 1 & 0,059 & 1 & 0,6 & 1,1 & 6,3 & 0,8 & 1,7 & 2,5 & 0,8 \\ 1 & 0,056 & 1 & 0,6 & 1,1 & 6,3 & 0,8 & 1,6 & 2,4 & 0,8 \\ 1 & 0,032 & 1 & 0,6 & 0,6 & 6,3 & 0,8 & 1,1 & 2,0 & 0,7 \\ 1 & 0,031 & 1 & 0,6 & 0,6 & 6,3 & 0,8 & 1,1 & 2,0 & 0,7 \\ 1 & 0,025 & 1 & 0,6 & 0,5 & 6,3 & 0,8 & 1,0 & 1,8 & 0,6 \\ 1 & 0,024 & 1 & 0,6 & 0,5 & 6,3 & 0,8 & 1,0 & 1,8 & 0,6 \\ 1 & 0,023 & 1 & 0,6 & 0,4 & 6,3 & 0,8 & 1,0 & 1,8 & 0,6 \\ 1 & 0,017 & 1 & 0,6 & 0,3 & 6,3 & 0,8 & 0,9 & 1,7 & 0,6 \\ 1 & 0,017 & 1 & 0,6 & 0,3 & 6,3 & 0,8 & 0,9 & 1,7 & 0,6 \\ 1 & 0,015 & 1 & 0,6 & 0,3 & 6,3 & 0,8 & 0,8 & 1,7 & 0,6 \\ 1 & 0,013 & 1 & 0,6 & 0,2 & 6,3 & 0,8 & 0,8 & 1,6 & 0,5 \\ 1 & 0,013 & 1 & 0,6 & 0,2 & 6,3 & 0,8 & 0,8 & 1,6 & 0,5 \\ 1 & 0,010 & 1 & 0,6 & 0,2 & 6,3 & 0,8 & 0,7 & 1,6 & 0,5 \\ 1 & 0,010 & 1 & 0,6 & 0,2 & 6,3 & 0,8 & 0,7 & 1,6 & 0,5 \\ 1 & 0,010 & 1 & 0,6 & 0,2 & 6,3 & 0,8 & 0,7 & 1,6 & 0,5 \\ 1 & 0,009 & 1 & 0,6 & 0,2 & 6,3 & 0,8 & 0,7 & 1,5 & 0,5 \\ 1 & 0,009 & 1 & 0,6 & 0,2 & 6,3 & 0,8 & 0,7 & 1,5 & 0,5 \\ 1 & 0,007 & 1 & 0,6 & 0,1 & 6,3 & 0,8 & 0,7 & 1,5 & 0,5 \\ 1 & 0,007 & 1 & 0,6 & 0,1 & 6,3 & 0,8 & 0,7 & 1,5 & 0,5\end{array}$

Legenda: $\mathrm{NI}=$ número de indivíduos; $\mathrm{AB}\left(\mathrm{m}^{2}\right)=$ área basal; $\mathrm{NP}=$ número de parcelas; $\mathrm{DR}=$ densidade relativa; $\mathrm{DoR}=$ dominância relativa; $\mathrm{FA}=$ freqüência absoluta; $\mathrm{FR}=$ freqüência relativa; IVC = índice de valor de cobertura; IVI = índice de valor de importância; \% IVI = porcentagem de índice de valor de importância. 
Tabela 3. Parâmetros fitossociológicos das espécies arbóreo-arbustivas de campo sujo na Floresta Nacional de Silvânia, Goiás, Brasil.

\begin{tabular}{|c|c|c|c|c|c|c|c|c|c|c|}
\hline Espécie & NI & $\mathbf{A B}\left(\mathrm{m}^{2}\right)$ & NP & DR & DoR & FA & FR & IVC & IVI & $\%$ IVI \\
\hline Styrax ferrugineus & 16 & 0,593 & 7 & 21,9 & 30,3 & 70,0 & 13,2 & 52,2 & 65,4 & 21,80 \\
\hline Piptocarpha rotundifolia & 8 & 0,280 & 4 & 11,0 & 14,3 & 40,0 & 7,5 & 25,3 & 32,8 & 10,94 \\
\hline Erythroxylum deciduum & 4 & 0,084 & 4 & 5,5 & 4,3 & 40,0 & 7,5 & 9,8 & 17,3 & 5,78 \\
\hline Kielmeyera coriacea & 5 & 0,054 & 4 & 6,8 & 2,8 & 40,0 & 7,5 & 9,6 & 17,2 & 5,73 \\
\hline Tabebuia aurea & 3 & 0,164 & 2 & 4,1 & 8,4 & 20,0 & 3,8 & 12,5 & 16,3 & 5,42 \\
\hline Mortos & 4 & 0,092 & 3 & 5,5 & 4,7 & 30,0 & 5,7 & 10,2 & 15,9 & 5,29 \\
\hline Byrsonima pachyphylla & 3 & 0,066 & 3 & 4,1 & 3,4 & 30,0 & 5,7 & 7,5 & 13,1 & 4,38 \\
\hline Dimorphandra mollis & 3 & 0,076 & 2 & 4,1 & 3,9 & 20,0 & 3,8 & 8,0 & 11,8 & 3,93 \\
\hline Handroanthus ochraceus & 3 & 0,035 & 2 & 4,1 & 1,8 & 20,0 & 3,8 & 5,9 & 9,7 & 3,22 \\
\hline Guapira noxia & 2 & 0,072 & 1 & 2,7 & 3,7 & 10,0 & 1,9 & 6,4 & 8,3 & 2,77 \\
\hline Schefflera macrocarpa & 2 & 0,031 & 2 & 2,7 & 1,6 & 20,0 & 3,8 & 4,3 & 8,1 & 2,70 \\
\hline Terminalia argentea & 2 & 0,028 & 2 & 2,7 & 1,4 & 20,0 & 3,8 & 4,2 & 8,0 & 2,65 \\
\hline Sapindaceae sp. & 2 & 0,021 & 2 & 2,7 & 1,1 & 20,0 & 3,8 & 3,9 & 7,6 & 2,54 \\
\hline Diospyros hispida & 2 & 0,056 & 1 & 2,7 & 2,9 & 10,0 & 1,9 & 5,6 & 7,5 & 2,50 \\
\hline Qualea parviflora & 1 & 0,067 & 1 & 1,4 & 3,4 & 10,0 & 1,9 & 4,8 & 6,7 & 2,23 \\
\hline Sclerolobium paniculatum & 1 & 0,058 & 1 & 1,4 & 3,0 & 10,0 & 1,9 & 4,4 & 6,3 & 2,09 \\
\hline Pseudobombax longiflorum & 1 & 0,041 & 1 & 1,4 & 2,1 & 10,0 & 1,9 & 3,5 & 5,4 & 1,79 \\
\hline Roupala montana & 1 & 0,028 & 1 & 1,4 & 1,5 & 10,0 & 1,9 & 2,8 & 4,7 & 1,57 \\
\hline Erythroxylum sp. & 1 & 0,018 & 1 & 1,4 & 0,9 & 10,0 & 1,9 & 2,3 & 4,2 & 1,40 \\
\hline Vochysia sp. & 1 & 0,015 & 1,0 & 1,4 & 0,8 & 10,0 & 1,9 & 2,2 & 4,0 & 1,35 \\
\hline Indeterminada & 1 & 0,012 & 1 & 1,4 & 0,6 & 10,0 & 1,9 & 2,0 & 3,9 & 1,30 \\
\hline Qualea grandiflora & 1 & 0,012 & 1 & 1,4 & 0,6 & 10,0 & 1,9 & 2,0 & 3,9 & 1,30 \\
\hline Ocotea sp. & 1 & 0,010 & 1 & 1,4 & 0,6 & 10,0 & 1,9 & 1,9 & 3,8 & 1,27 \\
\hline Leptolobium dasycarpum & 1 & 0,008 & 1 & 1,4 & 0,4 & 10,0 & 1,9 & 1,8 & 3,7 & 1,23 \\
\hline Byrsonima coccolobifolia & 1 & 0,008 & 1 & 1,4 & 0,4 & 10,0 & 1,9 & 1,8 & 3,7 & 1,22 \\
\hline Davilla elliptica & 1 & 0,008 & 1 & 1,4 & 0,4 & 10,0 & 1,9 & 1,8 & 3,7 & 1,22 \\
\hline Tibouchina sp. & 1 & 0,008 & 1 & 1,4 & 0,4 & 10,0 & 1,9 & 1,8 & 3,7 & 1,22 \\
\hline Psidium sp. & 1 & 0,007 & 1 & 1,4 & 0,4 & 10,0 & 1,9 & 1,7 & 3,6 & 1,21 \\
\hline
\end{tabular}

Legenda: $\mathrm{NI}=$ número de indivíduos; $\mathrm{AB}\left(\mathrm{m}^{2}\right)$ = área basal; $\mathrm{NP}=$ número de parcelas; $\mathrm{DR}=$ densidade relativa; $\mathrm{DoR}=$ dominância relativa; $\mathrm{FA}=$ freqüência absoluta; $\mathrm{FR}=$ freqüência relativa; IVC = índice de valor de cobertura; IVI = índice de valor de importância; \%IVI = porcentagem de índice de valor de importância. 
O diâmetro ao nível do solo encontrado para o campo sujo variou entre 4,8 e $17,5 \mathrm{~cm}$ (média de $8 \pm 2,6 \mathrm{~cm}$ ), já para o cerrado s.r. variou entre 4,8 e 30,2 (média de $8,7 \pm 4,2 \mathrm{~cm}$ ). O maior diâmetro médio foi representado por um indivíduo de Qualea parviflora Mart. para o campo sujo $(14,6 \mathrm{~cm})$ e de Sclerolobium paniculatum Vog. para o cerrado s.r. $(30,2 \mathrm{~cm})$. Considerando-se as classes de diâmetro para análise dos padrões de estratificação (Tabela 4), a frequência de indivíduos entre as classes foi diferentemente significativa $\left(\mathrm{X}^{2}=9,5, \mathrm{df}=3, \mathrm{p}<0,05\right)$, sendo que a maioria dos indivíduos do campo sujo esteve presente na classe $4(>10 \mathrm{~cm})$ com $34,3 \%$ e na classe $1(<6 \mathrm{~cm})$ com 32,9\%. Já no cerrado s.r., as classes não diferiram significativamente quanto ao número de indivíduos, como pode ser visto nas classes 1 $(<6 \mathrm{~cm})$ com $27,5 \%$, classe $4(>10 \mathrm{~cm})$ com $28,1 \%$ e classe $2(6,1-8 \mathrm{~cm})$ com $26,4 \%\left(X^{2}=4,7, d f=3, p=0,18\right)$. Isso indica que o campo sujo passa por um estágio de sucessão com muitos indivíduos bem desenvolvidos e muitos em estágio inicial de desenvolvimento. Já no cerrado s.r., a relativa equitabilidade entre as classes de diâmetro indica um estágio de desenvolvimento clímax. Muitos estudos para esse tipo de comunidade (savânica) têm mostrado um padrão onde a maioria dos indivíduos está nas classes de diâmetro mais baixas (chamado padrão de $\mathrm{J}$ invertido), indicando, por exemplo, que essas comunidades estão passando por um processo de regeneração (Assunção \& Felfili 2004; Moura et al. 2007).

Tabela 4. Distribuição dos indivíduos nas diferentes classes de diâmetro em fitofisionomias de campo sujo e cerrado sentido restrito na Floresta Nacional de Silvânia, Goiás, Brasil.

\begin{tabular}{lcccc}
\hline Classes & $\begin{array}{c}\text { N de } \\
\text { indivíduos no } \\
\text { campo sujo }\end{array}$ & $\begin{array}{c}\text { \% de } \\
\text { indivíduos } \\
\text { no campo } \\
\text { sujo }\end{array}$ & $\begin{array}{c}\text { N de } \\
\text { Indivíduos no } \\
\text { cerrado }\end{array}$ & $\begin{array}{c}\% \text { de indivíduos } \\
\text { no cerrado }\end{array}$ \\
\hline Classe 1 $(<6 \mathrm{~cm})$ & 24 & 32,9 & 49 & 27,5 \\
Classe 2 $(6,1-8 \mathrm{~cm})$ & 15 & 20,5 & 47 & 26,4 \\
Classe 3 $(8,1-10 \mathrm{~cm})$ & 9 & 12,3 & 32 & 18,0 \\
Classe 4 $(>10 \mathrm{~cm})$ & 25 & 34,3 & 50 & 28,1 \\
Total & 73 & 100 & 178 & 100 \\
\hline
\end{tabular}

A altura dos indivíduos no campo sujo variou entre 0,9 e $6 \mathrm{~m}$ (média de 2,47 $\pm 0,9 \mathrm{~m}$ ). No cerrado s.r. a altura variou entre 0,5 e $6 \mathrm{~m}$ (média de $2,4 \pm 1,07 \mathrm{~m}$ ). As maiores alturas foram representadas por um indivíduo de Schefflera macrocarpa (Cham. \& Schltdl.) Frodin no campo sujo e de S. paniculatum, Q. grandiflora, Terminalia argentea Mart. e Caryocar brasiliense Cambess. no cerrado s.r., todos com $6 \mathrm{~m}$ de altura. Considerando-se as quatro classes de altura que foram estabelecidas para a análise da estratificação vertical (Tabela 5), no campo sujo, a diferença entre as classes foi significativa $\left(X^{2}=26,4, d f=3, p<0,001\right)$, sendo que a maioria dos indivíduos $(41,1 \%)$ teve altura menor que $2 \mathrm{~m}$, estando presentes na 
classe 1. Da mesma forma a frequência de indivíduos entre as classes de altura diferiu no cerrado s.r. $\left(\mathrm{X}^{2}=74,0, \mathrm{df}=3, \mathrm{p}<0,001\right)$, onde $42,1 \%$ dos indivíduos estiveram na classe 2, entre 2,1 e $3 \mathrm{~m}$ de altura. Apesar dessas diferenças, a altura média entre as duas fitofisionomias variou pouco. Esses resultados indicam que ambas as vegetações apresentam uma grande proporção de indivíduos jovens (Assunção \& Felfili 2004).

$\mathrm{O}$ índice de Shannon $\left(\mathrm{H}^{\prime}\right)$ encontrado para o cerrado s.r. foi de 3,48 nats/indivíduo e com valores similares ocorreu nas 11 áreas de cerrado s.r. estudadas por Felfili et al. (1997), que variou entre 3,11 e 3,56 nats/indivíduo. O índice de equabilidade de Pielou $\left(\mathrm{J}^{\prime}\right)$ encontrado foi de 0,87. Para o campo sujo foram encontrados $\mathrm{H}^{\prime}=2,91$ e $\mathbf{J}^{\prime}=0,87$, valores também altos para esse tipo de vegetação (ver Felfili et al. 2004). Estes índices evidenciam uma alta diversidade de espécies para a área em estudo.

Quando comparadas quanto à composição e estrutura, as duas fitofisionomias estudadas apresentam semelhanças. A densidade absoluta foi de 730 indivíduos/ha no campo sujo e 1125 indivíduos/ha no cerrado s.r., já a área basal total foi de $5,32 \mathrm{~m}^{2} / \mathrm{ha}$ e $1,95 \mathrm{~m}^{2} / \mathrm{ha}$, no cerrado s.r. e no campo sujo, respectivamente. Quanto à área basal, S. ferrugineus, por exemplo, que foi a mais representativa no campo sujo, foi a terceira no cerrado s.r. O mesmo acontece com $K$. coriacea, $P$. rotundifolia, Byrsonima pachyphylla A.Juss. e Tabebuia aurea (Silva Manso) Benth. \& Hook. f. ex $\mathrm{S}$. Moore, importantes em ambas as formações vegetais.

Tabela 5. Distribuição dos indivíduos nas diferentes classes de altura em fitofisionomias de campo sujo e cerrado sentido restrito na Floresta Nacional de Silvânia, Goiás, Brasil.

\begin{tabular}{lcccc}
\hline Classes & $\begin{array}{c}\text { N de } \\
\text { indivíduos } \\
\text { no campo } \\
\text { sujo }\end{array}$ & $\begin{array}{c}\text { \% de } \\
\text { indivíduos no } \\
\text { campo sujo }\end{array}$ & $\begin{array}{c}\text { N de } \\
\text { Indivíduos no } \\
\text { cerrado }\end{array}$ & $\begin{array}{c}\% \text { de } \\
\text { indivíduos no } \\
\text { cerrado }\end{array}$ \\
\hline Classe 1 $(<2 \mathrm{~m})$ & 30 & 41,1 & 70 & 39,3 \\
Classe 2 $(2,1-3 \mathrm{~m})$ & 25 & 34,2 & 75 & 42,1 \\
Classe 3 $(3,1-4,9 \mathrm{~m})$ & 17 & 23,3 & 25 & 14,0 \\
Classe 4 $(>5 \mathrm{~m})$ & 1 & 1,4 & 8 & 4,5 \\
Total & 73 & 100 & 178 & 100 \\
\hline
\end{tabular}

Comparado à Serra Negra, GO, que tem 97 espécies listadas (Felfili \& Silva Júnior 2005), o cerrado s.r. da FLONA-Silvânia apresenta uma riqueza relativamente baixa (52 spp.) (Tabela 6). Por outro lado, para o local foi registrado um índice de Shannon elevado $(3,48)$ e o maior valor de equitabilidade $(0,87)$ entre os estudos comparados (Tabela 6). 
Tabela 6. Parâmetros das comunidades lenhosas observadas em diferentes tipos de cerrado sentido restrito, em várias localidades do Distrito Federal, Goiás e Mato Grosso, Centro-Oeste do Brasil.

\begin{tabular}{|c|c|c|c|c|c|c|c|c|}
\hline \multirow{2}{*}{ Local } & \multirow{2}{*}{ Método } & \multirow{2}{*}{ Fitofisionomia } & \multicolumn{5}{|c|}{ Parâmetros } & \multirow{2}{*}{ Fonte } \\
\hline & & & $\mathrm{A}$ & B & $\mathrm{C}$ & $\mathrm{D}$ & $\mathrm{E}$ & \\
\hline $\begin{array}{l}\text { Floresta Nacional } \\
\text { de Silvânia, GO }\end{array}$ & Parcela & Cerrado típico & 52 & 1.125 & 5,32 & 3,48 & 0,87 & $\begin{array}{l}\text { Presente } \\
\text { estudo }\end{array}$ \\
\hline $\begin{array}{l}\text { Ambiental do } \\
\text { Paranoá, DF }\end{array}$ & Parcela & Cerrado típico & 54 & 882 & 9,53 & 3,41 & - & 2004 \\
\hline $\begin{array}{c}\text { Área de Proteção } \\
\text { Ambiental Gama- } \\
\text { Cabeça de Veado, } \\
\text { DF }\end{array}$ & Parcela & Cerrado típico & 66 & 1.396 & 10,64 & 3,56 & - & $\begin{array}{l}\text { Felfili et } \\
\text { al. } 1993\end{array}$ \\
\hline $\begin{array}{c}\text { Estação Ecológica } \\
\text { de Águas } \\
\text { Emendadas, DF }\end{array}$ & Parcela & Cerrado típico & 72 & 1.396 & 10,76 & 3,62 & - & $\begin{array}{l}\text { Felfili et } \\
\text { al. } 1993\end{array}$ \\
\hline $\begin{array}{c}\text { Jardim Botânico } \\
\text { de Brasília, DF } \\
\text { (interflúvio) }\end{array}$ & Parcela & Cerrado típico & 53 & 1.219 & 8,6 & 3,16 & - & $\begin{array}{l}\text { Fonseca \& } \\
\text { Silva } \\
\text { Júnior } \\
2004\end{array}$ \\
\hline $\begin{array}{c}\text { Jardim Botânico } \\
\text { de Brasília, DF } \\
\text { (vale) }\end{array}$ & Parcela & Cerrado típico & 54 & 970 & 6,7 & 3,4 & - & $\begin{array}{l}\text { Fonseca \& } \\
\text { Silva } \\
\text { Júnior } \\
2004\end{array}$ \\
\hline $\begin{array}{l}\text { Município de } \\
\text { Água Boa, MT }\end{array}$ & Parcela & Cerrado típico & 80 & 995 & 7,5 & 3,69 & 0,84 & $\begin{array}{l}\text { Felfili et } \\
\text { al. } 2002 \\
\text { Felfili \& }\end{array}$ \\
\hline $\begin{array}{c}\text { Município de Alto } \\
\text { Paraíso, GO }\end{array}$ & Parcela & Cerrado típico & 92 & 944 & 8,05 & 3,46 & 0,76 & $\begin{array}{l}\text { Silva } \\
\text { Júnior } \\
2005\end{array}$ \\
\hline $\begin{array}{l}\text { Município de } \\
\text { Brasília, DF }\end{array}$ & Parcela & $\begin{array}{l}\text { Cerrado } \\
\text { rupestre }\end{array}$ & 51 & 631 & 3,67 & 3,08 & - & $\begin{array}{l}\text { Amaral et } \\
\text { al. } 2006\end{array}$ \\
\hline $\begin{array}{l}\text { Município de } \\
\text { Canarana, MT }\end{array}$ & Parcela & Cerrado típico & 88 & 1.285 & 9,5 & 3,78 & 0,84 & $\begin{array}{l}\text { Nogueira } \\
\text { et al. } 2001 \\
\text { Felfili \& }\end{array}$ \\
\hline $\begin{array}{c}\text { Município de } \\
\text { Serra Negra, GO }\end{array}$ & Parcela & Cerrado típico & 97 & 1.271 & 9,55 & 3,57 & 0,78 & $\begin{array}{l}\text { Silva } \\
\text { Júnior } \\
2005\end{array}$ \\
\hline $\begin{array}{l}\text { Município de } \\
\text { Silvânia, GO }\end{array}$ & Parcela & Cerrado típico & 68 & 1.348 & 11,3 & 3,31 & - & $\begin{array}{l}\text { Felfili et } \\
\text { al. } 1993\end{array}$ \\
\hline $\begin{array}{c}\text { Norte de Goiás e } \\
\text { Sul do Tocantins, } \\
\text { GO/TO }\end{array}$ & Parcela & $\begin{array}{l}\text { Cerrado } \\
\text { rupestre }\end{array}$ & 87 & 836 & 8,44 & 2,87 & 0,78 & $\begin{array}{l}\text { Felfili \& } \\
\text { Fagg } 2007\end{array}$ \\
\hline $\begin{array}{c}\text { Parque Estadual } \\
\text { da Serra de } \\
\text { Caldas Novas, } \\
\text { GO }\end{array}$ & $\begin{array}{l}\text { Quadra } \\
\text { nte }\end{array}$ & Cerrado típico & 67 & 1.907 & - & - & - & $\begin{array}{l}\text { Silva et al. } \\
2002\end{array}$ \\
\hline
\end{tabular}




\begin{tabular}{|c|c|c|c|c|c|c|c|c|}
\hline $\begin{array}{l}\text { Tabela } 6 . \\
\text { Continuação }\end{array}$ & & & & & & & & \\
\hline $\begin{array}{l}\text { Parque Estadual } \\
\text { dos Pireneus, GO }\end{array}$ & Parcela & $\begin{array}{l}\text { Cerrado } \\
\text { rupestre }\end{array}$ & 56 & 507 & 3,91 & 3,33 & 0,82 & $\begin{array}{l}\text { Moura et } \\
\text { al. } 2007\end{array}$ \\
\hline $\begin{array}{c}\text { Parque Nacional } \\
\text { da Chapada dos } \\
\text { Veadeiros, GO }\end{array}$ & Parcela & Cerrado típico & 85 & 1.110 & 8,92 & 3,49 & 0,78 & $\begin{array}{l}\text { Felfili \& } \\
\text { Silva- } \\
\text { Júnior } \\
2005\end{array}$ \\
\hline $\begin{array}{l}\text { Parque Nacional } \\
\text { de Brasília, DF }\end{array}$ & Parcela & Cerrado típico & 55 & 1036 & 8,32 & 3,34 & - & $\begin{array}{l}\text { Felfili et } \\
\text { al. } 1993\end{array}$ \\
\hline $\begin{array}{c}\text { Reserva } \\
\text { Ecológica do } \\
\text { IBGE, DF }\end{array}$ & Parcela & $\begin{array}{l}\text { Cerrado } \\
\text { denso }\end{array}$ & 63 & 1.964 & 13,28 & 3,53 & 0,85 & $\begin{array}{l}\text { Andrade } \\
\text { et al. } 2002\end{array}$ \\
\hline $\begin{array}{c}\text { Serra da Mesa, } \\
\text { GO }\end{array}$ & Parcela & Cerrado típico & 91 & 1.019 & 9,17 & 3,56 & 0,79 & $\begin{array}{l}\text { Felfili \& } \\
\text { Silva } \\
\text { Júnior } \\
2005\end{array}$ \\
\hline
\end{tabular}

Legenda: $\mathrm{A}=$ riqueza (número de espécies); $\mathrm{B}=$ densidade (número de indivíduos por hectare); $\mathrm{C}=$ área basal $\left(\mathrm{m}^{2}\right) ; \mathrm{D}=$ índice de diversidade de Shannon $\left(\mathrm{H}^{\prime}\right) ; \mathrm{E}=$ índice de equabilidade de Pielou (J').

Esse é o primeiro estudo sobre a estrutura florística da FLONA-Silvânia e já demonstra resultados interessantes, pois mostram que as comunidades lenhosas de campo sujo e cerrado s.r., apesar de não apresentarem um número grande de espécies, abrigam uma alta equitabilidade e diversidade. Além disso, esse trabalho representa a primeira contribuição sobre os campos sujos da região, uma vez que, levantamentos nesse tipo de vegetação no Centro-Oeste são escassos, e.g. (Tannus \& Assis 2004). De uma maneira geral esse trabalho é importante por elucidar parâmetros da comunidade vegetal que permitem, por exemplo, conhecer a estrutura da vegetação da região, bem como espécies da flora que podem ser importantes para a manutenção de outras populações de plantas e animais. Entretanto, sugerimos que novos estudos sobre fenologia e ecologia das espécies, bem como, estudos florísticos e fitossociológicos mais longos e englobando as demais fitofisionomias, são ainda necessários e encorajados para um maior conhecimento da flora da unidade.

\section{Agradecimentos}

Ao ICM-Bio pela oportunidade de desenvolver o trabalho na unidade, aos funcionários da FLONA-Silvânia e pesquisadores do IPAAC pelo apoio logístico nos trabalhos de campo.

\section{Referências}

Andrade,L.A.Z.; Felfili, J.M.;Violatti, L.2002. Fitossociologia de uma área de cerrado denso da RECOR-IBGE. Acta Botanica Brasilica 16: 225-240. 
Assunção, S.L.; Felfili, J.M. 2004. Fitossociologia de um fragmento de cerrado sensu stricto na APA do Paranoá, DF, Brasil. Acta Botanica Brasilica 18: 903-909.

Cunha, H.F.; Ferreira, A.A.; Brandão, D. 2007. Composição e fragmentação do Cerrado em Goiás usando Sistema de Informação Geográfica (SIG). Boletim Goiano de Geografia 27: 139-152.

Felfili, J.M.; Nogueira, P.E.; Silva Júnior, M.C.; Marimon, B.S.; Delitti, W.B.C. 2002. Composição florística e fitossociologia do cerrado sentido restrito no município de Água Boa - MT. Acta Botanica Brasilica 16: 103-112.

Felfili, J.M.; Silva Júnior, M.C. 2005. Diversidade alfa e beta no cerrado sensu stricto, Distrito Federal, Goiás, Minas Gerais e Bahia. In: Scariot, A.; SousaSilva, J.C.; Felfili, J.M. (Orgs.). Cerrado: ecologia, biodiversidade e conservação. Brasília: Ministério do Meio Ambiente. p. 143-154.

Felfili, J.M.; Silva Júnior, M.C.; Rezende, A.V.; Nogueira, P.E.; Walter, B.M.T.; Felfili, M.C.; Silva, M.A.; Encinas, J.I. 1997. Comparação florística e fitossociológica do cerrado nas Chapadas Pratinha e dos Veadeiros. In: Leite, L.L.; Saito, C.H. (Eds.). Contribuição ao conhecimento ecológico do Cerrado. Brasília: Editora da Universidade de Brasília. p. 6-11.

Felfili, J.M.; Silva Júnior, M.C.; Sevilha, A.C.;Fagg, C.W.; Walter, B.M.T.; Nogueira, P.E.; Rezende, A.V. 2004. Diversity, floristic and structural patterns of cerrado vegetation in Central Brazil. Plant Ecology 175: 37-46.

Felfili, J.M.; Venturoli, F. 2000. Comunicações Técnicas Florestais: Tópicos de análise de vegetação. 2(2): 1-34.

Fonseca, M.S.; Silva Júnior, M.C. 2004. Fitossociologia e similaridade florística entre trechos de Cerrado sentido restrito em interflúvio e em vale no Jardim Botânico de Brasília, DF. Acta Botanica Brasilica 18: 19-29.

Francener, A.; Hall, C.F.;Porfírio-Júnior, E.D.; Araújo, W.S. 2012. Flora fanerogâmica da Floresta Nacional de Silvânia, Goiás, Brasil. Enciclopédia Biosfera 8: 12631277.

Klink, C.A.; Machado, R.B. 2005. Conservation of Brazilian Cerrado. Conservation Biology 19: 707-713.

Lorenzi, H. 1992. Árvores brasileiras: manual de identificação e cultivo de plantas arbóreas nativas do Brasil. Nova Odessa: Plantarum. 352 p.

Mendonça, R.C.; Felfili, J.M.; Walter, B.M.T.; Silva Júnior, M.C.; Rezende, A.B.; Filgueiras, T.S.; Nogueira, P.E.; Fagg, C.W. 2008. Flora vascular do Bioma Cerrado: checklist com 12.356 espécies. In: Sano, S.M.; Almeida, S.P.; Ribeiro, J.F. (Orgs.). Cerrado: Ecologia e Flora. Brasília: Embrapa Cerrados. p. 213228. v.2.

Morais, A.R.; Bastos, R.P.; Vieira, R.; Signorelli, L. 2012. Herpetofauna da Floresta Nacional de Silvânia, um remanescente de cerrado no Brasil Central. Neotropical Biology and Conservation 7: 114-121.

Moura, I.O.; Gomes-Klein, V.L.; Felfili, J.M. 2007. Fitossociologia da comunidade lenhosa de uma área de cerrado rupestre no Parque Estadual dos Pireneus, Pirenópolis, Goiás. Revista de Biologia Neotropical 4: 83-100. 
Myers, N.; Mittermeier, R.A.; Mittermeier, C.G.; Fonseca, G.A.B.; Kent, J. 2000. Biodiversity hotspots for conservation priorities. Nature 403: 853-858.

Oliveira-Filho, A.T.; Ratter, J.A. 2002. Vegetation physionomies and wood flora of the Cerrado Biome. In: Oliveira, P.S.; Marquis, R.J. (Eds.). The Cerrados of Brazil: ecology and natural history of a neotropical savanna. New York: Columbia University Press. p. 91-120.

Ratter, J.A.; Ribeiro, J.F.; Bridgewater, S. 1997. The Brazilian Cerrado Vegetation and Threats to its Biodiversity. Annals of Botany 80: 223-230.

Ribeiro, J.F.; Walter, B.M.T. 1998. Fitofisionomias do bioma Cerrado, p.89-168. In: S.M. Sano \& S.P. Almeida (Eds.). Cerrado: ambiente e flora. Planaltina: Embrapa/CPAC. p. 89-168.

Silva, L.O.; Costa, D.A.; Filho K.E.S.; Brandão, D. 2002. Levantamento florístico e fitossociológico em duas áreas de cerrado sensu stricto no Parque Estadual da Serra de Caldas Novas, Goiás. Acta Botanica Brasilica 16: 43-53.

Silva Júnior, M.C. 2005. 100 Árvores do Cerrado: guia de campo. Brasília: Rede de Sementes do Cerrado. v. 1.278 p.

Tannus, J.L.S.; Assis, M.A. 2004. Composição de espécies vasculares de campo sujo e campo úmido em área de cerrado, Itirapina - SP, Brasil. Revista Brasileira de Botânica 27: 489-506.

The Angiosperm Phylogeny Group. 2009. An update of the Angiosperm Phylogeny Group classification for the orders and families of flowering plants: APG III. Botanical Journal of the Linnean Society 161: 105-121. 